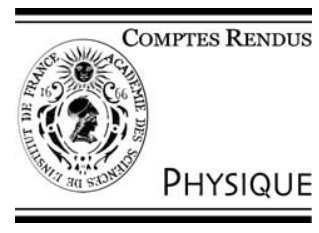

The Cosmic Microwave Background/Le rayonnement fossile à $3 \mathrm{~K}$

\title{
The MAXIMA experiment: latest results and consistency tests
}

\author{
R. Stompor ${ }^{a, b}$, S. Hanany ${ }^{c}$, M.E. Abroe ${ }^{c}$, J. Borrill $^{\mathrm{a}, \mathrm{b}}$, P.G. Ferreira ${ }^{\mathrm{d}}$, \\ A.H. Jaffe ${ }^{\text {e }}$, B. Johnson ${ }^{\text {b }}$, A.T. Lee ${ }^{\text {f,g }}$, B. Rabii ${ }^{\text {b,f }}$, P.L. Richards ${ }^{\text {f,g }}$, \\ G. Smoot ${ }^{\text {b,f,g }}$, C. Winant ${ }^{\mathrm{f}}$, J.H.P. Wu ${ }^{\mathrm{h}}$ \\ a Computational Research Division, Lawrence Berkeley National Laboratory, Berkeley, CA 94720, USA \\ $\mathrm{b}$ Space Sciences Laboratory, University of California, Berkeley, CA 94720, USA \\ c School of Physics and Astronomy, University of Minnesota, Minneapolis, MN 55455, USA \\ $\mathrm{d}$ Astrophysics and Theoretical Physics, University of Oxford, Oxford, UK \\ e Astrophysics Group, Blackett Laboratory, Imperial College, London SW7 2BW, UK \\ ${ }^{\mathrm{f}}$ Department of Physics, University of California, Berkeley, CA 94720, USA \\ g Physics Division, Lawrence Berkeley National Laboratory, Berkeley, CA 94720, USA \\ h Department of Physics, National Taiwan University, Taipei, Taiwan
}

Presented by Guy Laval

\begin{abstract}
The MAXIMA cosmic microwave background anisotropy experiment had a significant impact on cosmology. Results from the program have played a significant role in determining the geometry of the universe, given strong supporting evidence to inflation, and, in combination with other astrophysical data, showed that the universe is filled with dark matter and energy. We present a subset of the internal consistency checks that were carried out on the MAXIMA-1 data prior to their release, which demonstrate that systematics errors were much smaller than statistical errors. We also discuss the MAXIMA-2 flight and data, compare the maps of MAXIMA-1 and -2 in areas where they overlap and show that the two independent experiments confirm each other. All of these results demonstrate that MAXIMA mapped the cosmic microwave background anisotropy with high accuracy. To cite this article: R. Stompor et al., C. R. Physique 4 (2003).
\end{abstract}

(C) 2003 Académie des sciences. Published by Elsevier SAS. All rights reserved.

\section{Résumé}

L'expérience MAXIMA : résultats et vérifications internes. L'expérience MAXIMA de mesure des anisotropies du fonds de rayonnement cosmique (CMB) a eu un impact significatif sur la cosmologie. Les résultats de ce programme ont joué un rôle important pour la détermination de la géométrie de l'univers, ont apporté de fortes indications expérimentales en faveur de l'inflation, et en combinaison avec d'autres données astrophysiques, ont montré que l'univers est constitué principalement de matière et d'énergie noires. Nous présentons une partie de la batterie de vérifications internes qui ont été appliquées aux données du vol MAXIMA-1 avant leur publication. Ces tests ont démontré que les erreurs systématiques étaient bien plus petites que les erreurs statistiques. Nous discutons également du vol et des données MAXIMA-2, comparons les cartes des vols MAXIMA-1 et -2 dans les régions de chevauchement et montrons que les deux expériences indépendantes se confirment mutuellement. Tous ces résultats démontrent que MAXIMA a cartographié les anisotropies du CMB avec une grande précision. Pour citer cet article : R. Stompor et al., C. R. Physique 4 (2003).

C 2003 Académie des sciences. Published by Elsevier SAS. All rights reserved.

Keywords: MAXIMA; Cosmic microwave background anisotropy; Cosmology; Early Universe

Mots-clés : MAXIMA ; Anisotropies du fonds de rayonnement cosmique ; Cosmologie ; Univers primordial 


\section{Introduction}

MAXIMA was a balloon-borne experiment that measured temperature fluctuations in the cosmic microwave background (CMB) radiation. The instrument consisted of a 16 element array of bolometric photometers operating between frequencies of 150 and 410 GHz. It flew twice in 1998 and 1999 from NASA's National Scientific Balloon Facility in Palestine, Texas and the two flights and their corresponding data sets have become known as MAXIMA-1 and MAXIMA-2, respectively. Direct results such as maps and power spectra, as well as derivative results, such as analysis techniques, cosmological implications, and assessment of foregrounds have been published in a number of papers. Jaffe et al. [1] have given a compilation of MAXIMA papers and since their paper several more papers have been written [2,3].

The MAXIMA-1 results had significant impact on cosmology. Together with the results from BOOMERanG [4], they showed conclusively that the geometry of the universe is close to flat [5], and supported the evidence of BOOMERanG [6] and DASI [7] for harmonic peaks in the power spectrum [8]. Fig. 1 illustrates this leap in information content. The top panel in the figure, which is a combination of all the CMB data prior to April 2000, shows that indications that the universe is flat were already evident in data of earlier experiments. The middle panel shows only the MAXIMA data as released in May 2000, shortly after the release of the BOOMERanG data. At the time of that first release the MAXIMA-1 data gave the highest resolution map of the $\mathrm{CMB}$ and had provided information over the broadest range of angular scales compared to any other experiment. To date MAXIMA has the highest reported sensitivity of any CMB photometer and the highest combined sensitivity of any CMB receiver. ${ }^{1}$ Hanany et al. [5] report photometer sensitivities as low as $80 \mu \mathrm{K} \cdot \sqrt{s}$ and a combined sensitivity of $46 \mu \mathrm{K} \cdot \sqrt{s}$ for the MAXIMA-1 data set.

Jaffe et al. [9] analyzed the accuracy with which the COBE-DMR, MAXIMA and BOOMERanG data constrain cosmological parameters when the datasets were analyzed separately and together. They found that the combination of COBEDMR and MAXIMA data constrained both the flatness of the universe and the spectral index of the power spectrum of spatial fluctuations $n$ to within $9 \%$ error (at $1 \sigma$ ). The inclusion of the BOOMERanG data improved the determination to within 6 and $9 \%$, respectively. The combination with other astrophysical data showed that the universe is dominated by dark matter and energy $[11,9]$. A year later, MAXIMA and BOOMERanG simultaneously released more of their data and DASI released new

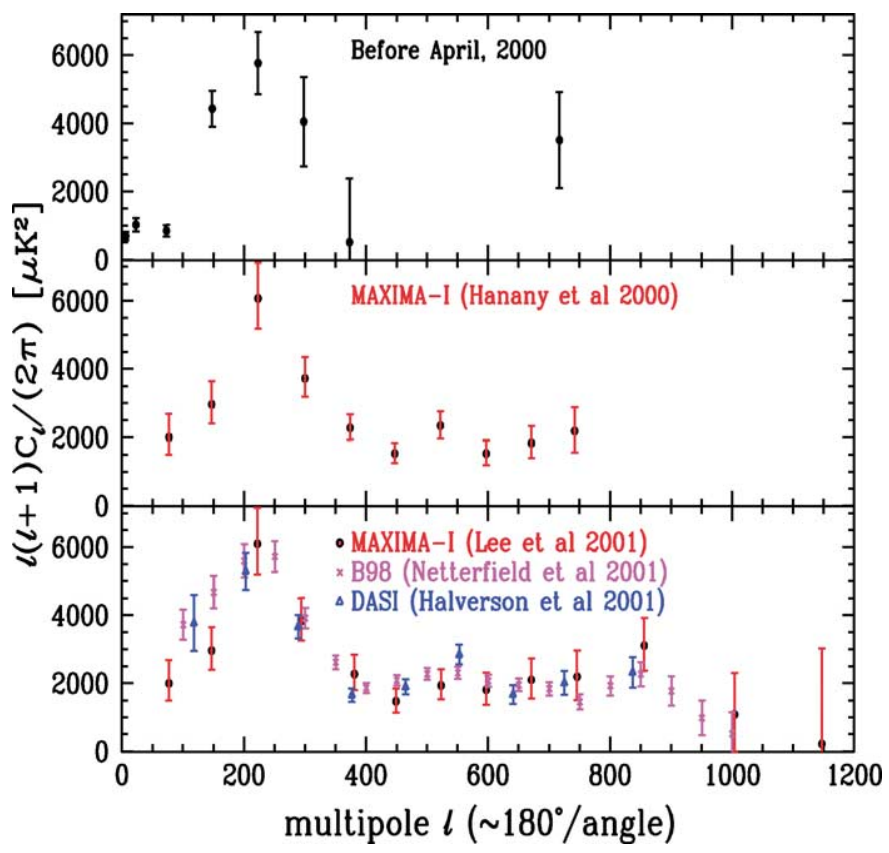

Fig. 1. A combination of all CMB data prior to the first release of the MAXIMA and BOOMERanG data (top panel, courtesy of A. Jaffe), the MAXIMA data alone in 2000 [5], and the 2001 data of MAXIMA, BOOMERanG, and DASI [6-8]. No calibration adjustments have been made to the power spectra.

\footnotetext{
1 'Receiver sensitivity' is defined as $\left[\sum_{i} 1 / \sigma_{i}^{2}\right]^{-1 / 2}$ where $\sigma_{i}$ is photometer sensitivity and the sum is over photometers from which combined data is published.
} 
results. The power spectrum results of MAXIMA essentially have not changed, but were extended to higher $\ell$ values. This 2001 collection of the data is shown in the bottom panel of Fig. 1. Where they overlapped, all three power spectra were remarkably consistent with each other. DASI and BOOMERanG gave higher signal-to-noise ratio on the harmonic acoustic peak structure while MAXIMA had a broader coverage in $\ell$.

The impact of all of these data was that within a span of one year cosmology radically changed. Inflation gained strong supporting evidence, the framework of a universe overwhelmingly dominated by unknown forms of dark matter and energy had been transformed from a debated possibility to an essentially accepted fact, and the precision of the determination of the cosmological parameters ushered what had been called the 'era of precision cosmology'. Subsequent data from other experiments and recently from WMAP have confirmed these conclusions and significantly improved the accuracy of the determination of all the cosmological parameters.

Before its release the MAXIMA-1 data were subjected to a battery of systematic tests to ensure its validity. The availability of data from several independent photometers as well as the high redundancy of the scan strategy provided multiple ways to cross-check the results and to ensure that the contribution of systematic errors was negligible. In Section 2 of this paper we present the results of many of these tests for the first time.

An even stronger systematic test is to cross-check the results against those from an independent experiment. We chose the scan region of MAXIMA-2 to partially overlap that of MAXIMA-1 to allow a detailed comparison. In Section 3 we give details of the MAXIMA-2 flight and data analysis and present some comparisons between the MAXIMA-1 and -2 data sets. A recent, more detailed analysis has shown conclusively that the MAXIMA-1, -2 and WMAP maps have detected the same spatial fluctuations in a common region of the sky [2].

\section{Systematic tests of the MAXIMA-1 data}

The MAXIMA instrument was reviewed in detail elsewhere [3,5,10]. The MAXIMA-1 map, power spectrum [5,8], and cosmological results $[11,12]$ are based on the analysis of the combination of data collected by the four photometers (three photometers for the Lee et al. paper [8]) that had the lowest noise equivalent temperatures (NET) [5]; hereafter we refer to them as $b 34, b 25, b 45$ and $b 33$, where $b$ stands for 'bolometer' and the two digits define the position of the bolometer in the $4 \times 4$ array. The first three detectors $(b 34, b 45$ and $b 25)$ operated at a frequency band centered on $150 \mathrm{GHz}$, and the forth (b33) at a frequency band centered on $240 \mathrm{GHz}$ (data from $b 33$ was not included in the results of Lee et al.).

We will discuss the following subset of systematics tests that have been carried out on the data:

- a comparison of the maps and power spectra that were calculated from the data of individual photometers (Section 2.1),

- a comparison of maps and power spectra of a given region of the sky, but for which the data was taken at different times during the flight (Section 2.2),

- a comparison of the power spectra of different regions of the map (Section 2.3).

We will also discuss the effects of pixelization and noise as they relate to the extraction of high $\ell$ information from the data (Section 2.4).

All the maps presented in this section were computed using a variation of the optimal maximum likelihood map-making using the circulant noise approach $[13,14]$. The maps have been pixelized using square pixels of 8 arcminutes on a side and unless otherwise noted are made from the data of all four photometers. When estimating a power spectrum we deconvolved a circular top-hat pixel with an area equal to that of the pixel [15]. 'Sum maps' are noise-weighted co-addition of constituent maps, and 'difference maps' are half of the unweighted difference of the pixels common to both maps. Power spectra were computed using the quadratic estimator approach [16,17] with the MADCAP implementation [18] and are presented with bins in spherical harmonic number $\ell$ of width $\Delta \ell=75$. The spectral bin amplitudes have been decorrelated [17]. The theoretical power spectrum shown for reference in dotted line in some of the figures is the best fit cosmological model to the MAXIMA-1 data as given by Stompor et al. [12].

\subsection{Data of individual photometers}

\subsubsection{Maps}

Of the four photometers used for CMB data, the noise level of $b 34$ was the lowest, achieving an NET of $80 \mu \mathrm{K} \cdot \sqrt{s}$ for most of the flight, and $b 33$ had the highest NET of $120 \mu \mathrm{K} \cdot \sqrt{s}$. It is therefore interesting to compare the maps and power spectra derived from the data of these photometers. In either case the time domain noise properties were almost stationary throughout the entire $\mathrm{CMB}$ measurement, not exceeding an end-to-end change in the white noise level of 10-20\% in the most extreme cases. Maps made from the data of $b 34$ and $b 33$ are shown in Fig. 2 and show similar structure throughout the map but more 

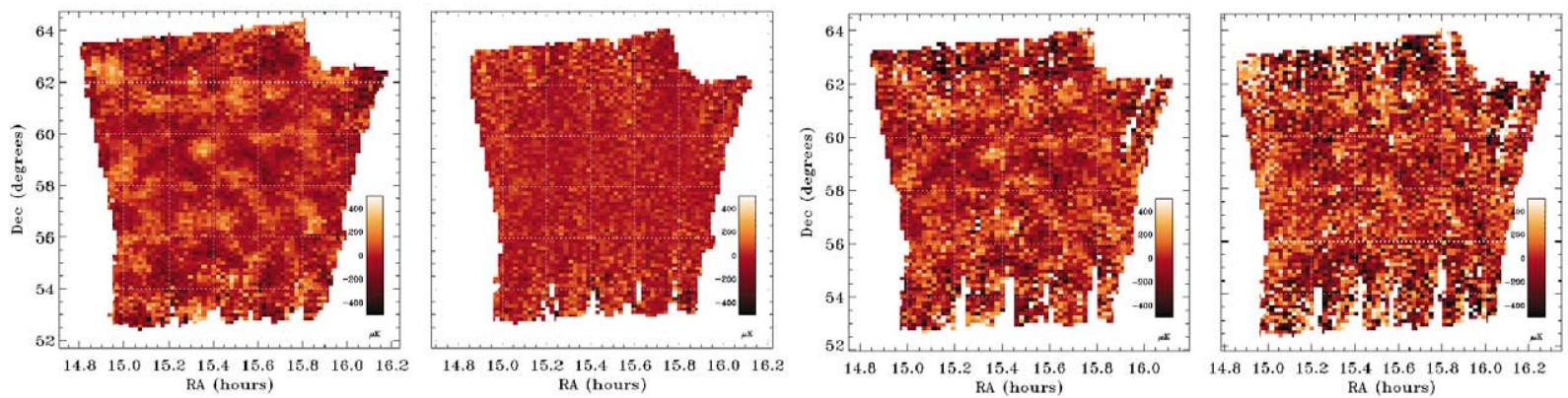

Fig. 2. Left to right: (i) the final map made from the data from all four detectors combined; (ii) the map of the pixel noise computed from the difference of two maps where each is made from the combination of data from only two detectors (i.e., $b 34+b 45$ and $b 25+b 33$ ); (iii) map from the data of $b 34$ only; and (iv) map from the data of $b 33$ only. Similar spatial fluctuations are present in all of the three maps that have a CMB signal and are absent in the map of the noise.

predominantly in the low-noise central part of the maps. The same sky structure is also readily discernible in the map made by combining the data of all four detectors, but the structure disappears in the four-detector $((b 34+b 33)-(b 45+b 25))$ difference map. This visual impression is expressed quantitatively using the following statistics:

- the $\chi^{2}$ statistic,

$$
\chi^{2}(\mathbf{m}) \equiv \mathbf{m}^{\mathrm{T}} \mathcal{N}^{-1} \mathbf{m}, \quad \kappa(\mathbf{m}) \equiv \frac{\left(\chi^{2}(\mathbf{m})-n_{\mathrm{DOF}}\right)}{\sqrt{2 n_{\mathrm{DOF}}}},
$$

where $\mathbf{m}$ and $\mathcal{N}$ denote a map and a pixel-pixel noise correlation matrix, respectively. The statistic has $n_{\text {DOF }}$ effective degrees of freedom, which are assumed to be equal to the difference between the number of pixels and the low $\ell$-modes that are removed from the map prior to the analysis. Assuming Gaussian noise, the coefficient $\kappa$ quantifies the distance in units of standard deviation between the computed value of $\chi^{2}$ and the value expected if no sky signal was present in the map;

- the 'null buster' statistic $v$ [19],

$$
v(\mathbf{m}) \equiv \frac{\mathbf{m}^{\mathrm{T}} \mathcal{N}^{-1} \mathcal{S} \mathcal{N}^{-1} \mathbf{m}-\operatorname{Tr}\left[\mathcal{N}^{-1} \mathcal{S}\right]}{\left\{2 \operatorname{Tr}\left[\mathcal{N}^{-1} \mathcal{S} \mathcal{N}^{-1} \mathcal{S}\right]\right\}^{1 / 2}},
$$

where $\mathcal{S}$ is an arbitrary matrix, which is equal to the signal correlation matrix computed for the best fit MAXIMA-1 power spectrum smoothed with a Gaussian beam of 10 arcminutes full-width at half maximum and an axially symmetric (approximate) pixel window function [19]. This statistic determines the number of standard deviations at which a given map $\mathbf{m}$ is inconsistent with a hypothesis of only having noise, given that the signal is described by the correlation matrix $\mathcal{S}$ (and it is therefore analogous to the $\kappa$ statistic, with which it coincides if $\mathcal{S}=\mathcal{N}$ );

- the one-dimensional Kolmogorov-Smirnov (KS) test applied to noise-prewhitened maps [14] and defined as:

$$
\widehat{\mathbf{m}} \equiv \mathcal{F}^{-1 / 2} \mathbf{m}, \quad \text { where } \mathcal{F} \text { is assumed to be a symmetric matrix such as } \quad \mathcal{N} \equiv \mathcal{F}^{1 / 2} \mathcal{F}^{1 / 2} .
$$

For each map we compute a KS significance coefficient giving the confidence level at which the hypothesis that the prewhitened map has been randomly drawn from the Gaussian distribution with a unit variance can be accepted;

- the probability enhancement factor $\beta$ [20],

$$
\beta\left(\mathbf{m}_{i}, \mathbf{m}_{j}\right) \equiv \ln \left\{\frac{\mathcal{P}\left(\left[\begin{array}{l}
\mathbf{m}_{i} \\
\mathbf{m}_{j}
\end{array}\right] \mid\left[\begin{array}{cc}
\mathcal{C}_{i} & \mathcal{C}_{i j} \\
\mathcal{C}_{j i} & \mathcal{C}_{j}
\end{array}\right]\right)}{\mathcal{P}\left(\mathbf{m}_{i} \mid \mathcal{C}_{i}\right) \mathcal{P}\left(\mathbf{m}_{j} \mid \mathcal{C}_{j}\right)}\right\}
$$

where the matrix $\mathcal{C}_{i}$ describes the CMB signal correlation matrix computed for a map $\mathbf{m}_{i}$, and $\mathcal{C}_{i j}$ is the signal crosscorrelation matrix for maps $\mathbf{m}_{i}$ and $\mathbf{m}_{j}$. In our case both are computed assuming the MAXIMA- 1 best fit power spectrum smoothed with the antenna beam and the pixel window function for the null-buster statistic. The quantity $\mathcal{P}\left(\mathbf{m}_{i} \mid \mathcal{C}_{i}\right)$ represents the probability distribution of realizations of maps with signal correlations given by $\mathcal{C}_{i}$ and noise correlations given by $\mathcal{N}_{i}$; we assume that the probability distribution is a multi-variate Gaussian. We assign a statistical significance to this statistic by computing its mean and variance either under the assumption of no correlation or the assumption of full correlation of the sky signal in both maps [20,21]. We denote these value $\beta_{0}\left(\mathbf{m}_{i}, \mathbf{m}_{j}\right)$ and $\beta_{\infty}\left(\mathbf{m}_{i}, \mathbf{m}_{j}\right)$ respectively. 
Due to the small size of the MAXIMA-1 maps the very low- $\ell$ content of the maps may not be reliable so one may not want to include it in the tests described here. Therefore in the case of the $\chi^{2}$, 'null-buster' and KS statistics we 'weighted out' [17] all the $\ell$-modes with $\ell \leqslant 35$ by replacing the inverse noise correlation matrices $\mathcal{N}^{-1}$ by

$$
\mathcal{N}^{-1} \longrightarrow \mathcal{N}^{-1}-\left(\mathcal{N}^{-1} \mathcal{B}\right)^{\mathrm{T}}\left[\mathcal{B}^{\mathrm{T}} \mathcal{N}^{-1} \mathcal{B}\right]^{-1}\left(\mathcal{N}^{-1} \mathcal{B}\right), \quad \text { where } \mathcal{B}_{i k} \equiv \sum_{j} \psi_{j}^{i}\left[\psi_{j}^{k}\right]^{\mathrm{T}}
$$

and $\psi$ constitute a set of linearly independent pixel vectors spanning the same space as all the spherical harmonics with $\ell \leqslant 35$; $\psi_{j}^{i}$ is the $i$-th pixel component of the vector $\psi_{j}$. This correction corresponds to assigning 'infinite' noise to the spatial modes described by the functions $\psi[17,14]$. Consequently these modes do not contribute to the final results of any of the statistics. For the probability enhancement factor we have applied an analogous correction to the inverse (signal + noise) correlation matrix, $\mathcal{S}+\mathcal{N}$. For the sky patches considered here we usually find that there are only $\simeq 55$ independent modes (and hence vectors $\psi$ ) out of a total of 1296 spherical harmonics with $\ell \leqslant 35$. We have also found that although the particular values of the statistical tests depend on whether the modes with $\ell \leqslant 35$ are rejected or not, the overall conclusions remain essentially unchanged.

The results of these tests as applied to various pairs of maps are given in Table 1. They confirm the visual agreement between the maps that were produced from the data of different detectors. The absolute values of $\kappa$ and $v$ computed for the difference maps are usually $\lesssim 1$, and always less then 2 , which is to be interpreted as a 'better than $2 \sigma$ ' agreement. For the probability enhancement factor, the value of $\beta$ always agrees with the expected value of $\beta_{0}$ within the quoted ' $1 \sigma$ ' uncertainty and always disagrees by more than ' $6 \sigma$ ' (and usually $\sim 15-20 \sigma$ ) with the appropriate $\beta_{\infty}$. The latter values are expected for $\beta$ if there is no correlation between a given pair of maps. Note that both the null-buster statistic and the probability enhancement factor depend on the choice of the signal power spectrum. However, we have found that if we adopt a flat power spectrum rather than the best-fit spectrum chosen above then the numbers computed for these statistics change by no more than $10-20 \%$ and their statistical interpretation remains the same [19].

We have also applied the $\chi^{2}$, null-buster and KS statistics to the single detector maps. The results are collected in Table 2 and show that a strong signal is detected in all cases. In the case of the null-buster test, the numbers computed here can be compared with those obtained for the Saskatoon and QMAP experiments which are 21 and 40, respectively [22]. It is clear that according to this statistic there is more information content in a map made from a single detector of MAXIMA-1 than there is in the final maps produced by either of those experiments. When contrasted with the values obtained for the difference maps, these numbers can be viewed as a demonstration of the sensitivity of the tests. However it is important to bear in mind that the noise level and correlations are different in the two-detector difference maps than in any single detector map.

Table 1

Results of statistical tests that were applied to the differences of pairs of maps (columns 3,4 and 5) that were produced from the data of photometers listed in columns 1 and 2, the probability enhancement factor test (column 6) applied to the same pairs of maps, and the expected average and 68\% (' $1 \sigma^{\prime}$ ) confidence ranges under the hypothesis of perfect (column 7) or lack of (column 8) correlations of the signal in both maps. Columns 3, 4 and 5 have results for the $\chi^{2}$, Kolmogorov-Smirnov and null-buster statistics, respectively, and show very good consistency with the hypothesis that the difference maps contain no sky signal

\begin{tabular}{ccccrcrc}
\hline $\mathbf{m}_{1}$ & $\mathbf{m}_{2}$ & $\kappa$ & KS $(\%)$ & $v$ & $\beta$ & $\beta_{0}$ & $\beta_{\infty}$ \\
\hline$b 34$ & $b 45$ & -1.9 & 28 & -0.4 & -180 & $-166 \pm 14$ & $-2540 \pm 200$ \\
$b 34$ & $b 25$ & -0.06 & 44 & -0.8 & -198 & $-184 \pm 15$ & $-2560 \pm 210$ \\
$b 34$ & $b 33$ & -0.2 & 49 & -0.5 & -146 & $-142 \pm 14$ & $-2640 \pm 440$ \\
$b 45$ & $b 25$ & -0.7 & 91 & -0.3 & -172 & $-171 \pm 15$ & $-2440 \pm 100$ \\
$b 33$ & $b 45$ & 0.1 & 22 & 0.6 & -128 & $-129 \pm 13$ & $-2450 \pm 120$ \\
$b 25$ & $b 33$ & -0.02 & 84 & -0.9 & -143 & $-139 \pm 13$ & $-2460 \pm 130$ \\
$b 34+b 33$ & $b 45+b 25$ & 0.3 & 90 & -0.6 & -292 & $-281 \pm 18$ & $-2970 \pm 230$ \\
CMB1 & CMB2 & -1.6 & 81 & 0.1 & -168 & $-195 \pm 15$ & $-1860 \pm 130$ \\
\hline
\end{tabular}

Table 2

Results of the same statistical tests as shown in Table 1 but applied to single photometer maps. Since these maps do contain sky signal, contrasting these results with those in Table 1 demonstrates the sensitivity of each statistic to the presence of sky signal in the map. Zeros in the case of the KS statistics stand for numbers less than $10^{-7}$

\begin{tabular}{lccccccccc}
\hline & $b 34$ & $b 45$ & $b 25$ & $b 33$ & $b 34+b 45$ & $b 25+b 33$ & CMB1 & CMB2 & ALL \\
\hline$\kappa(\mathbf{m})$ & 16 & 11 & 20 & 7 & 30 & 28 & 33 & 24 & 69 \\
$\mathrm{KS}(\mathbf{m})(\%)$ & 0 & 0.5 & 0 & 1 & 0 & 0 & 0 & 0 & 0 \\
$\nu(\mathbf{m})$ & 70 & 60 & 100 & 35 & 130 & 135 & 140 & 140 & 317 \\
\hline
\end{tabular}




\subsubsection{Power spectra}

The power spectra for each of the detectors individually and combined (Fig. 3) are consistent throughout the entire $\ell$ range, with the scatter in the estimated bin power increasing at the higher and noisier $\ell$ bins. The error bars plotted here reflect minimally correlated statistical uncertainty only, and exclude any fully correlated systematic uncertainties. Such systematic uncertainties could come from an overall misestimation of the calibration, which has the effect of renormalizing the entire power spectrum, or from beam reconstruction uncertainty, which is important predominantly at high $\ell$. The calibration uncertainty is about $8 \%$ in power for the data of any single photometer and we have conservatively assumed a combined calibration uncertainty of $8 \%$ for the combination of all photometers. The beam reconstruction uncertainty of MAXIMA-1 has been investigated in great detail by $\mathrm{Wu}$ et al. [15] and Lee et al. [8].

\subsubsection{Difference maps}

Differencing two maps of the same patch of the sky is a sensitive method of searching for systematic problems in the data. Power spectra of such difference maps - unlike the 'single number' statistics of Section 2.1.1 - may not only detect a problem but also locate the angular scale at which it occurs, thereby providing a useful diagnostic.

From the four single detector MAXIMA-1 maps we form six distinct, although not independent, difference maps; the power spectra of these difference maps are shown in Fig. 4. A $\chi^{2}$ with a null model gives values of $\simeq 1$ per degree of freedom for all differences. The only points deviating from zero by more than $2 \sigma$ are found at the very low- $\ell$ end of the power spectra. This is not surprising given the difficulty of estimating the lowest frequency noise modes in the time domain [14], which dominate the noise contribution on large angular scales. If we interpret any residual power as an estimate of a systematic error, we find that the magnitude of such an error is much smaller than the statistical uncertainty in the power in the corresponding bins.

The slight excess of positive detections over negative at high $\ell$ (which may appear to be a trend, but in fact does not continue to yet higher $\ell$, see for example the right panel of Fig. 8) is most likely the residual of sky signal that persists in the difference maps due to the somewhat different beams of the various detectors. Although such a signal is expected to be rather small, it is amplified by the deconvolution of the beam and pixel window function in the power spectrum estimation. The error budget of
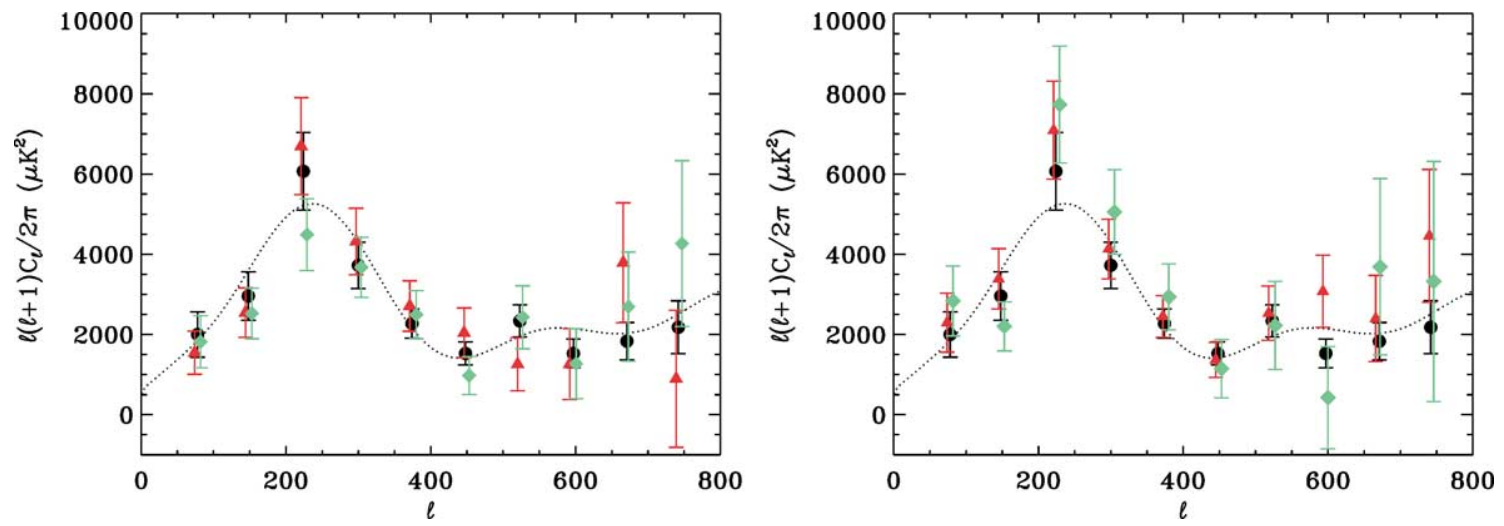

Fig. 3. Angular power spectra using the data of $b 34$ (b25) in triangles and of $b 45$ (b33) in diamonds in the left (right) panel. The power spectrum from the combined data from all four detectors (circles) [5] is also shown for comparison. In each bin the triangles and diamonds have been displaced slightly from the true central values (shown by the filled circles) to make the figure readable. The dotted curves is a best fit cosmology to the MAXIMA-1 results [12].
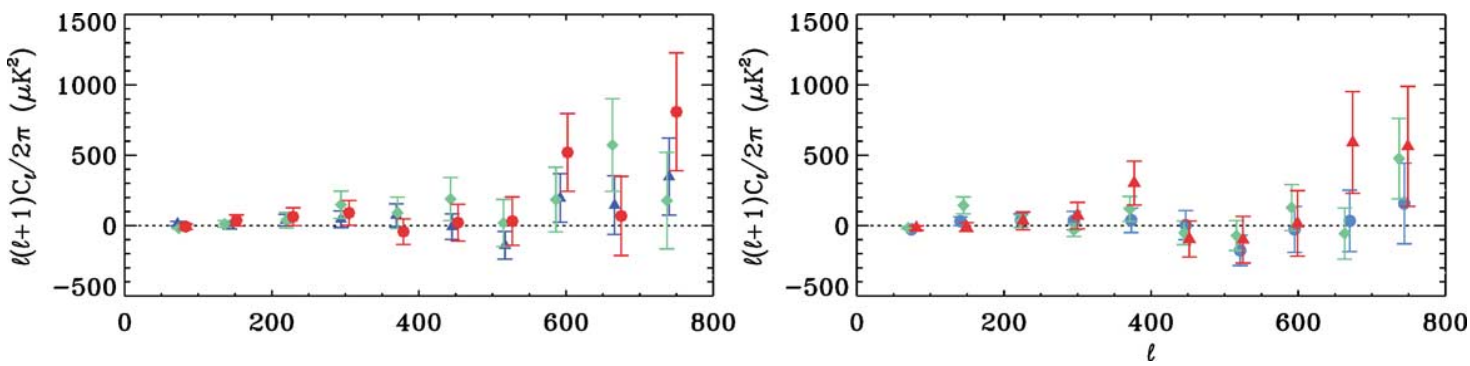

Fig. 4. Angular power spectra of the single detector difference maps. The left panel shows the three combinations excluding, and the right panel including, the $b 45$ photometer. 
the final MAXIMA-1 spectrum [5] includes the effect of differences of beams between different detectors but the effect is not included when calculating difference spectra such as shown in Fig. 4.

\subsection{Temporal comparison}

During the 1998 flight of MAXIMA each photometer observed the same patch of the sky twice, with an approximately 90 minute gap between observations. This provides a natural division of the data into two parts, which we call CMB1 and CMB2. These scans are the two green shaded areas in the left panel of Fig. 5, which are oriented at an angle of about 20 degrees to each other. Because of the time lag between the scans the maps of CMB1 and CMB2 may have different systematic errors and it is valuable to compare them. The maps made from the combination of the data from four photometers are shown in the two right panels of Fig. 5. We can clearly see that the structure is generally well replicated in each map. The visual impression is confirmed when we calculate the statistics of Section 2.1.1 to compare the maps, and also when we calculate the corresponding power spectra, which agree well both with one another and with the 'canonical' MAXIMA-1 spectrum; see the left panel in Fig. 6. Some concern might be raised by the bright spot in the CMB2 map (at RA $\simeq 15.7$ hours and DEC $\simeq 57$ degree) which has no counterpart in CMB1. We do not expect this feature to have any bearing on the final results, although we have failed to single out an unambiguous source for the difference, or even to determine its statistical significance. This is probably an artifact of the map-making algorithm due to poor cross-linking in this region. This suspicion is supported by the observation that no feature of this sort is found in the better cross-linked map combining the data from both scans. Moreover, applying the power spectrum analysis to maps with the pixels corresponding to this feature removed shows no significant change in the results.

\subsection{Spatial comparison}

An interesting test of the data is to compute and compare the power spectra of sub-maps of the entire map. Such sub-map spectra should agree to within the sampling and noise variances. The disadvantage of this approach is that because of pixelpixel noise and sky signal correlations, the interpretation of differences between the spectra obtained is not straightforward. Furthermore the uncertainties in the sub-map spectra rapidly grow as the number of pixels decreases, making comparisons between small sub-maps meaningless. Here we investigate two halving subdivisions of the full map - left versus right and top versus bottom. These spectra are shown in the right panel of Fig. 6 and are in good agreement.

\subsection{The High e regime}

The first release of the MAXIMA-1 data [5] included information only up to $\ell=785$ because more time and computational effort was required to ensure that all systematic errors have been analyzed thoroughly for the higher $\ell$ regime. In the second release [8] a subset of the data from the first release was analyzed to give information up to $\ell=1200$. Here we discuss how this subset of the data was chosen.

\subsubsection{Spatial cut}

Pixelization of the maps introduces an extra smoothing of the underlying CMB signal on very small scales. Applying an appropriate window function to compensate for the smoothing (as described earlier) assumes an unrealistic perfect sampling
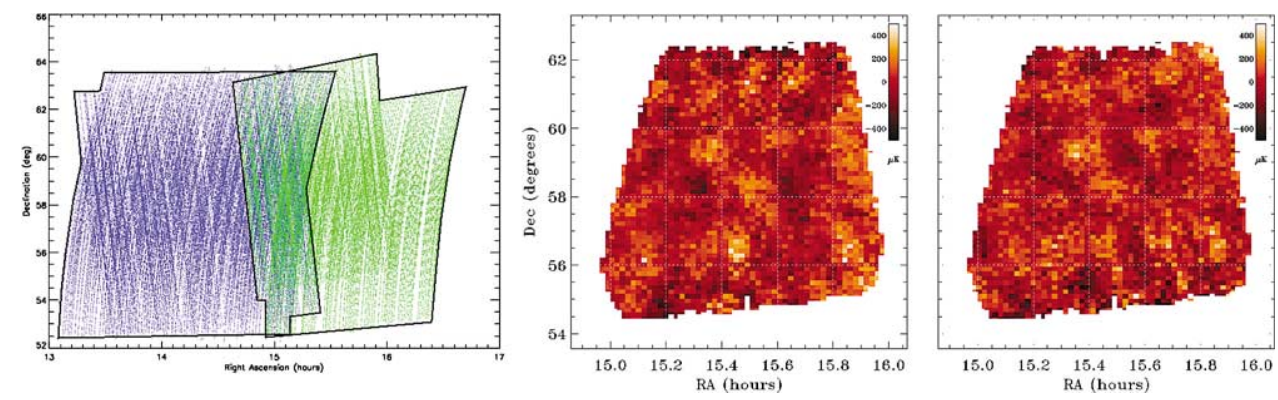

Fig. 5. Left: the areas of the sky scanned by MAXIMA-1 (green) and MAXIMA-2 (blue). Each of the areas was scanned with a 'CMB1' and 'CMB2' distinct scans that were taken at different times and that have a relative angle of about 25 degrees. Each point in the plot represents a pointing of the telescope averaged over $\sim 100$ msec. The MAXIMA-2 area overlaps about 50 square degrees of the area of MAXIMA-1 providing an important systematic test. Right: Maps of the MAXIMA-1 CMB1 (left panel) and CMB2 (right) scans. Only the overlapping region of both scans is shown. 

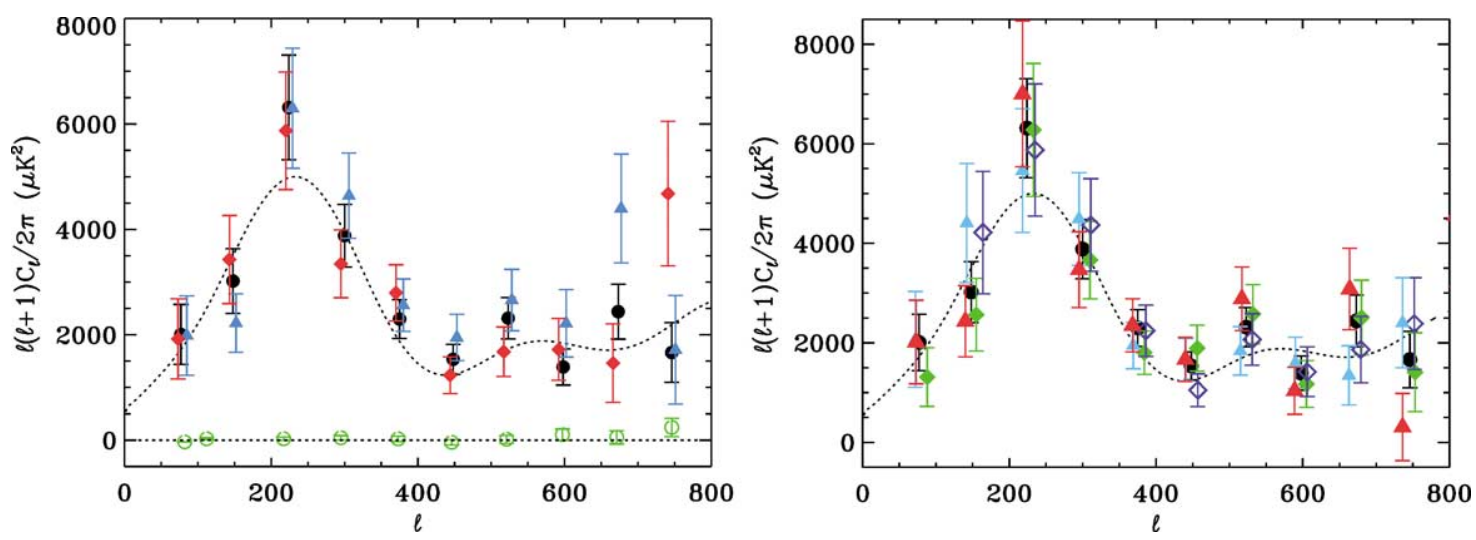

Fig. 6. Left: angular power spectra of the CMB1 (diamond) and CMB2 (triangle) scans using data from four detectors, the spectrum published by Hanany et al. [5] (filled circles) and spectrum of the difference map. Right: angular power spectra computed for the left and right halves of the map (filled and open diamonds, respectively) and the upper and lower halves (filled and open triangles) as well as the full map (filled circles). Each of the sub-maps contains only $\sim 3000$ pixels.

of every pixel in the map. In reality the smoothing introduced by the pixelization procedure is position (pixel) dependent, and difficult to deconvolve exactly from the final spectrum. One solution is to decrease the pixelization scale until the smoothing that it induces does not affect the spectrum in the range of $\ell$ of interest. However, this has to be weighed against the increased computational cost of analyzing maps with more pixels. Another solution is to use relatively big pixels but include only pixels that happen to be sampled very uniformly and for which the smoothing should be well characterized by the approximate window function.

We have chosen to use both approaches. For our high $\ell$ spectra we limited the analysis to those 8 arcminute pixels that had more than 100 samples and for which the variation in the number of observations in each quadrant of the pixel was less than $10 \%$. Because of the MAXIMA-1 scan pattern this choice corresponded to a spatial cut on the map where the 'central section' of the map was included and the edges excluded; the full map and the demarcation of the cut section are shown in Fig. 7. We also chose the pixel size to be 3 arcminutes so that the effect of extra smoothing at $\ell \sim 1000$ was less than $3 \%$, and clearly sub-dominant compared to the other statistical and systematic uncertainties. The left panel in Fig. 8 shows the power spectra of the entire MAXIMA-1 map pixelized with 8 arcminute pixels and with a deconvolution of an approximate pixel window function (as discussed in Section 2), only the central section pixelized with 8 arcminute pixels and with a deconvolution of the same window function, and the entire map pixelized with 3 arcminute pixels but with no deconvolution of a pixel window
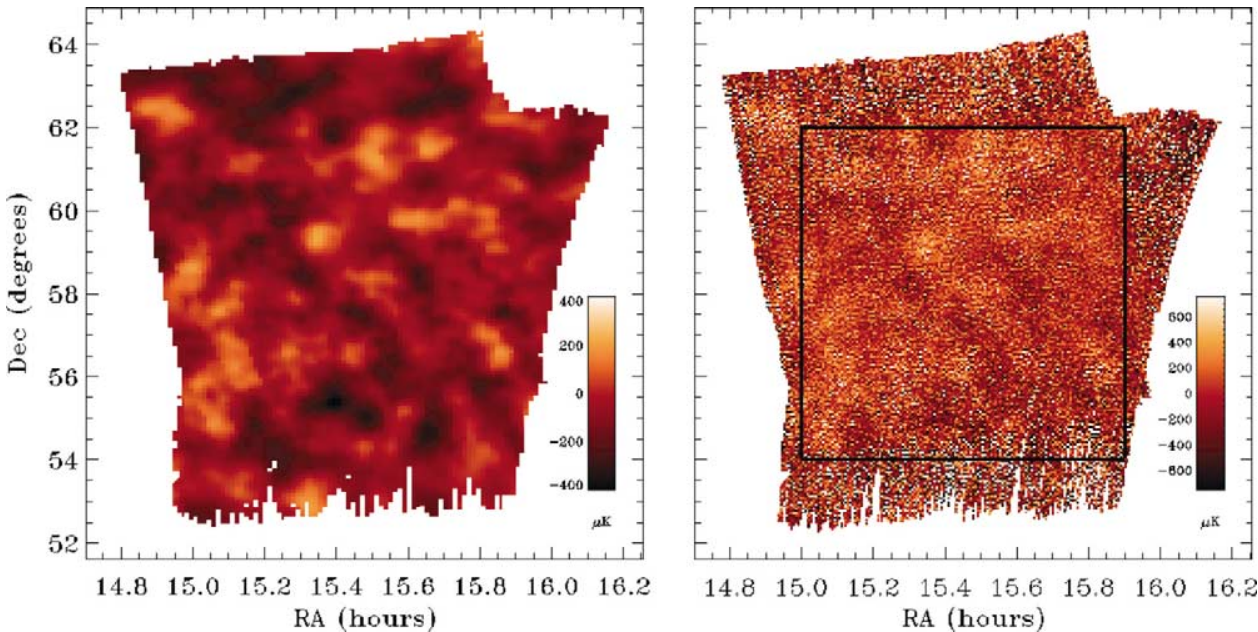

Fig. 7. The entire MAXIMA-1 map pixelized with 3 arcminute pixels and a demarcation of the region used to produce the high $\ell$ region of the power spectrum (right) and a Wiener filtered version of the map (left). The color stretches are $\pm 750 \mu \mathrm{K}$ and $\pm 400 \mu \mathrm{K}$, for the right and left panels, respectively. 

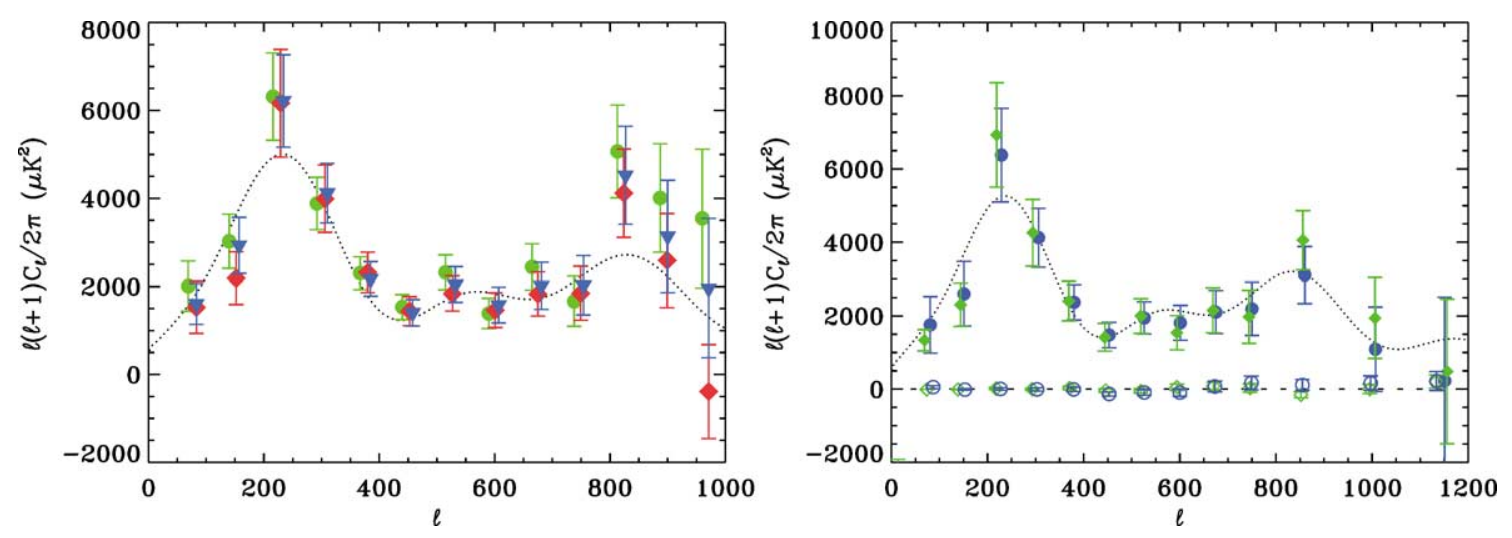

Fig. 8. Left: angular power spectra of a map with 8 arcminutes pixels (circles), only the central section of the map also pixelized with 8 arcminutes pixels (triangles) and the entire map with 3 arcminutes pixels, but with no deconvolution of a pixel window function (diamonds); see text. Right: power spectra of maps pixelized with 3 arcminutes and made using data from all four detectors (diamonds) and including only three detectors by excluding the data from $b 33$ (circles), and angular power spectra of difference maps of $(b 34+b 45)-(b 25+b 33)($ diamonds) and of $b 34-(b 45+b 25)$ (circles), both with a pixelization of 3 arcminutes.

function. The conclusions are that the spectrum at $\ell \gtrsim 800$ is sensitive to the details of the pixelization, and that the 8 arcminute pixelization overestimates the power at this $\ell$ range. Using only the well sampled 8 arcminute pixels reduces the discrepancy between power spectra from maps with an 8 and 3 arcminute pixelizations. The 3 arcminute power spectrum shown in the left panel of Fig. 8, for which we used the data of all four detectors, can be compared with the spectra shown in the right panel, which do include the deconvolution of an approximate ( 3 arcminute) window function, and use only the well sampled parts of the map (note that the binning at high $\ell$ is somewhat different between the two spectra).

\subsubsection{Data cut}

The major parasitic signal in the MAXIMA-1 time stream was related to the primary mirror modulation [5]. The amplitude of this signal, which was less than $\sim 100 \mu \mathrm{K}$ for $b 34, b 45$ and $b 25$, was comparable to the CMB signal, and therefore had to be removed. For $b 33$ the amplitude of the primary mirror synchronous signal was $\sim 300 \mu \mathrm{K}$ and the noise inherent to this determination was larger than for the $150 \mathrm{GHz}$ detectors. This higher amplitude and noise were inconsequential for the determination of the power spectrum at $\ell \lesssim 800$, as has been verified extensively in simulations and in various systematics tests (some of which have been presented earlier in this paper). However the effects of the synchronous signal for $b 33$ appeared non-negligible for the higher $\ell$ regime of the power spectrum. The power spectrum of a map made from data that included $b 33$ gave somewhat higher power at $\ell \geqslant 800$ compared with the power spectrum that excluded $b 33$, see the right panel of Fig. 8. No such difference was found when we excluded the data from any other photometer. Power spectra of difference maps of pairs of photometers that included $b 33$ showed small inconsistencies with a null spectrum (again at $\ell \geqslant 800$ ), but power spectra of difference maps of other pairs of photometers showed no such inconsistency. These inconsistencies were small - for example, they essentially disappeared in the difference maps made from combination of several photometers that included or excluded b33, see the right panel of Fig. 8 - and their origin appeared to be the mirror synchronous signal. Foreground contributions in the MAXIMA-1 region were sufficiently small and could not account for the observed inconsistencies. We therefore chose to exclude $b 33$ from the determination of the high $\ell$ spectrum.

\section{MAXIMA-2}

The 225 square degrees area of the sky that was scanned during the MAXIMA-2 flight in 1999 overlapped with 50 square degrees of the area scanned during MAXIMA-1 and was larger by about a factor of two, see Fig. 5. The expected detector performance and scan strategy were similar for the two flights. However, the data showed a somewhat higher level of systematic errors, which would have required more effort to understand and overcome. The collaboration decided to release only limited results that will facilitate the comparison between the MAXIMA-1 and MAXIMA-2 maps.

Similar to the data from MAXIMA-1, it was advantageous to analyze the MAXIMA-2 data that came from a subset of some of the most sensitive photometers. Those were $b 34, b 35, b 45$ and $b 25$ operating at $150 \mathrm{GHz}$. The operational parameters for these detectors including time constant, NET, band widths, and beam sizes are given in a paper by Rabii et al. [3]. Two of the 
$150 \mathrm{GHz}$ detectors gave an NET of $\sim 80 \mu \mathrm{K} \cdot \sqrt{s}$ and the NET for the combination of the MAXIMA-2 detectors was $43 \mu \mathrm{K} \cdot \sqrt{s}$, slightly out-performing the value of $46 \mu \mathrm{K} \cdot \sqrt{s}$ for the four best detectors of MAXIMA-1.

In addition to the CMB scan, the MAXIMA-2 flight included a calibration on the dipole and beam mapping using Mars. Dipole observations in MAXIMA-2 were conducted at float altitude (120 kft), unlike MAXIMA-1 in which they were started during ascent (70 kft). Because of this the MAXIMA-2 dipole analysis did not require any atmospheric subtraction as was done for the MAXIMA-1 data. During about 20\% of MAXIMA-2 CMB scan there were no detectable guide stars for pointing reconstruction. For this section stars were seen as rarely as once per 30 seconds and pointing reconstruction was based on the rate gyroscope. The total estimated pointing error during that time increased from 1 arcminute to 1.5 arcminutes RMS. Other aspects of the processing of the time ordered data, absolute calibration using the CMB dipole, relative calibration using a mmwave source internal to the receiver, beam shapes determination, and pointing reconstruction were analogous in all respects to
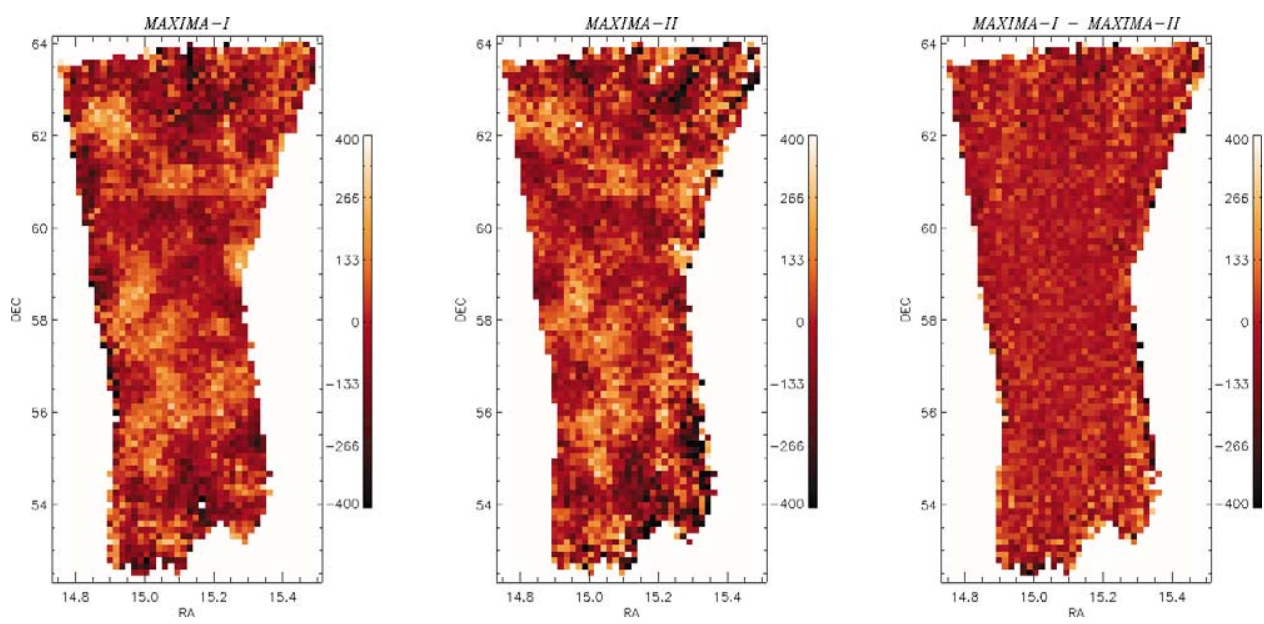

Fig. 9. Maps of the overlap region between the MAXIMA-1 (left) and MAXIMA-2 (middle) maps, and their difference (right). Abroe et al. [2] show a Wiener filtered version of these maps.

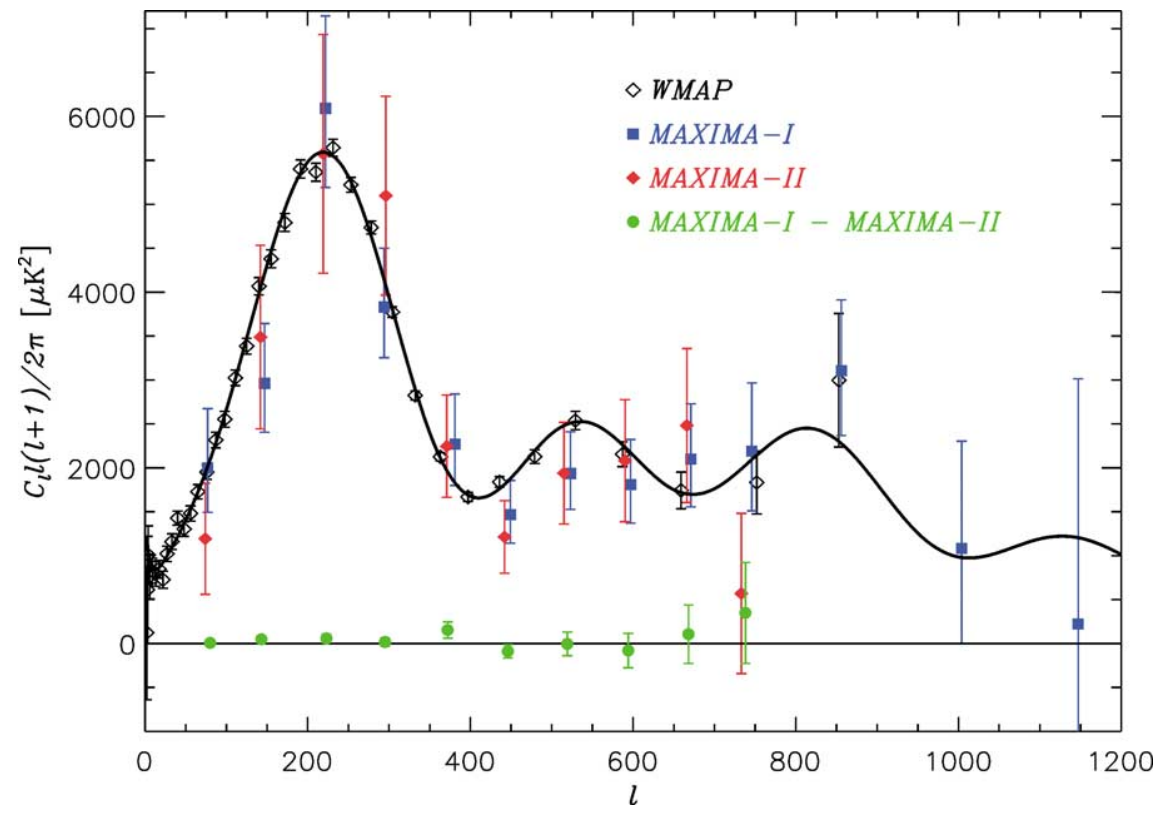

Fig. 10. Power spectra from the MAXIMA-1 data (squares) [8], the MAXIMA-2 data but only from the overlap region with MAXIMA-1 (filled diamonds), and a power spectrum of the difference map of the overlap region (circles) [2]. For reference we also show the data from WMAP (open diamonds) and the best fit cosmology to the WMAP data [23]. No calibration adjustments have been made to any of the spectra. 
those followed for the MAXIMA-1 analysis and which are described by Hanany et al. [5]. Rabii et al. [3] give more details about MAXIMA-2.

Estimating the maximum likelihood map also followed the prescription given by previous publications [5,8,14], but the characteristics of the data were somewhat different than that of MAXIMA-1. There were stronger drifts giving rise to a $1 / f^{2}$ characterization of the noise at low frequencies (compared with $1 / f$ with MAXIMA-1). The knee in the power spectrum between a $1 / f^{2}$ dependence and white noise occurred at a frequency of about $1 \mathrm{~Hz}$ (compared to $0.5 \mathrm{~Hz}$ with MAXIMA-1). A noise synchronous with the modulation of the primary mirror, which has also occurred with MAXIMA-1, had an amplitude of up to $500 \mu \mathrm{K}$ (as compared to less than $300 \mu \mathrm{K}$ for MAXIMA-1) and was not as stationary as in MAXIMA-1.

The maps made of the data of MAXIMA-2 and MAXIMA-1 in the areas where they overlap is shown in Fig. 9. Also shown is the difference map. To calculate the power spectra we pixelized the maps with 8 arcminutes square pixels giving 5972 and 2757 pixels for MAXIMA-1 and -2, respectively. The power spectra of MAXIMA-2 from this overlap region, the entire MAXIMA-1 data [5], and the spectrum of the difference map in the overlap region are shown in Fig. 10. The $\chi^{2}$ of a null spectrum model for the difference spectrum is 8 for 10 degrees of freedom. Abroe et al. [2] have correlated this MAXIMA-2 map with the maps from MAXIMA-1 and from WMAP $93 \mathrm{GHz}$ band and find a high degree of correlation, providing strong evidence that all three experiments have detected the same spatial temperature fluctuations in this region of the sky.

\section{Summary}

The MAXIMA results, together with other CMB results of that era, have radically changed cosmology. The combined COBE-DMR and MAXIMA results have constrained the flatness of the universe and the spectral index of the power spectrum of spatial fluctuations $n$ to unprecedented accuracy [11,9] and were consistent with data from BOOMERanG and DASI that showed peaks in the power spectrum at $\ell>250$. All of these advances together with other astrophysical data established the current model of cosmology: a flat universe that is overwhelmingly dominated by unknown forms of matter and energy.

In this paper we presented a subset of the systematic tests that were carried out on the MAXIMA-1 data before their release. We showed that systematic errors contributed negligibly to the final results thereby providing the necessary confidence for the cosmological interpretation of the data. More recently, the data have passed an even more stringent systematic test: comparison with independent data sets. The initial agreement of the power spectrum between MAXIMA-1, BOOMERanG, DASI and other experiments was reassuring, but the later maps of MAXIMA-2 (and WMAP, as shown by Abroe et al. [2]) give strong confidence that MAXIMA-1 has accurately mapped the cosmic microwave background anisotropy.

\section{Acknowledgements}

We greatfully acknowledge support from NASA's National Scientific Balloon Facility and NASA and NSF grants that have supported the MAXIMA program over the years. Computing resources for data analysis have been provided by the Minnesota Supercomputing Institute at the University of Minnesota and by the National Energy Research Computing Center, which is supported by the Office of Science of the U.S. Department of Energy under Contract No. DE-AC03-76SF00098. MA and RS acknowledge support from NASA's grant S-92548-F. SH acknowledges a Land-McKnight Professorship at the University of Minnesota.

\section{References}

[1] A.H. Jaffe, et al., in: New Astronomy Reviews, Proceedings of the CMBNET Meeting, Oxford, UK, 20-21 February, 2003, in press, astro-ph/0306504.

[2] M.E. Abroe, et al., Astrophys. J., submitted for publication, astro-ph/0308355.

[3] B. Rabii, et al., Astrophys. J., submitted for publication, astro-ph/0309414.

[4] P. De Bernardis, et al., Nature 404 (2000) 955.

[5] S. Hanany, et al., Astrophys. J. 545 (2000) L5.

[6] C.N. Netterfield, et al., Astrophys. J. 571 (2002) 604.

[7] N.W. Halverson, et al., Astrophys. J. 568 (2002) 38.

[8] A.T. Lee, et al., Astrophys. J. Lett. 561 (2001) L1.

[9] A.H. Jaffe, et al., Phys. Rev. Lett. 86 (2001) 3475.

[10] A.T. Lee, et al., Proceedings of the 3K Cosmology Meetings, 1999.

[11] A. Balbi, et al., Astrophys. J. 545 (2000) L5.

[12] R. Stompor, et al., Astrophys. J. Lett. 561 (2001) L1. 
[13] M. Tegmark, Phys. Rev. D 56 (1997) 4514.

[14] R. Stompor, et al., Phys. Rev. D 65 (2002) 022033.

[15] J.H.P. Wu, et al., Astrophys. J. Suppl. Ser. 132 (2001) 1.

[16] M. Tegmark, Phys. Rev. D 55 (1997) 5895.

[17] J.R. Bond, A.H. Jaffe, L. Knox, Phys. Rev. D 57 (1998) 2117.

[18] J.D. Borrill, 1999, astro-ph/9911389.

[19] M. Tegmark, Astrophys. J. Lett. 474 (1999) L77.

[20] L. Knox, J.R. Bond, A.H. Jaffe, M. Segal, D. Charbonneau, Phys. Rev. D 58 (1998) 083004, astro-ph/9903249.

[21] K. Coble, et al., Astrophys. J. 584 (2003) 585, astro-ph/0112506.

[22] Y. Xu, M. Tegmark, A. de Oliveira-Costa, Phys. Rev. D 65 (2002) 083002.

[23] D. Spergel, et al., Astrophys. J. Suppl. Ser. 148 (2003) 175. 